OPEN ACCESS

Edited by:

Rommel Chacon-Salinas, Instituto Politécnico Nacional (IPN),

Mexico

Reviewed by:

Luis Antonio Pérez-García, Autonomous University of San Luis

Potosí, Mexico

Eva Salinas,

Autonomous University of

Aguascalientes, Mexico Vanessa Lara

University of São Paulo, Brazil

*Correspondence:

Zuo-tao Zhao

zhaozuotaotao@163.com

Specialty section:

This article was submitted to Microbial Immunology, a section of the journal

Frontiers in Immunology

Received: 31 March 2021

Accepted: 19 May 2021

Published: 03 June 2021

Citation:

Yu M, Song X-t, Liu B, Luan T-t, Liao S-I and Zhao Z-t (2021) The Emerging Role of Mast Cells in Response to Fungal Infection.

Front. Immunol. 12:688659. doi: 10.3389/fimmu.2021.688659

\section{The Emerging Role of Mast Cells in Response to Fungal Infection}

\author{
Miao Yu ${ }^{1,2,3,4}$, Xiao-ting Song ${ }^{1,2,3}$, Bo Liu ${ }^{1,2,3}$, Ting-ting Luan ${ }^{1,2,3,4}$, Shuang-lu Liao ${ }^{1,2,3}$ \\ and Zuo-tao Zhao ${ }^{1,2,3^{*}}$
}

\begin{abstract}
${ }^{1}$ Department of Dermatology and Venerology, Peking University First Hospital, Beijing, China, ${ }^{2}$ Beijing Key Laboratory of Molecular Diagnosis on Dermatoses, Beijing, China, ${ }^{3}$ National Clinical Research Center for Skin and Immune Diseases, Beijing, China, ${ }^{4}$ Peking University School of Nursing, Beijing, China
\end{abstract}

Mast cells (MCs) have been considered as the core effector cells of allergic diseases. However, there are evidence suggesting that MCs are involved in the mechanisms of fungal infection. MCs are mostly located in the border between host and environment and thus may have easy contact with the external environmental pathogens. These cells express receptors which can recognize pathogen-associated molecular patterns such as Toll-like receptors (TLR2/4) and C-type Lectins receptors (Dectin-1/2). Currently, more and more data indicate that MCs can be interacted with some fungi (Candida albicans, Aspergillus fumigatus and Sporothrix schenckil). It is demonstrated that MCs can enhance immunity through triggered degranulation, secretion of cytokines and chemokines, neutrophil recruitment, or provision of extracellular DNA traps in response to the stimulation by fungi. In contrast, the involvement of MCs in some immune responses may lead to more severe symptoms, such as intestinal barrier function loss, development of allergic bronchial pulmonary aspergillosis and increased area of inflammatory in $S$. schenckii infection. This suggests that MCs and their relevant signaling pathways are potential treatment regimens to prevent the clinically unwanted consequences. However, it is not yet possible to make definitive statements about the role of MCs during fungal infection and/or pathomechanisms of fungal diseases. In our article, we aim to review the function of MCs in fungal infections from molecular mechanism to signaling pathways, and illustrate the role of MCs in some common host-fungi interactions.

Keywords: mast cell, fungi, pathogen-associated molecular patterns, Candida albicans, Aspergillus fumigatus, Sporothrix schenckii

\section{INTRODUCTION}

Fungal infections are a serious health problem all over the world. Currently, more than 300 million people suffer from severe fungal infections, and an estimated over 1.5 million people die from deep fungal infections each year (1). With broad-spectrum antibiotics used and the increase of immunodeficiency disease such as acquired immune deficiency syndrome, pathogenic fungi opportunistic infections showed a trend of rising sharply (2-4). Although early diagnosis and the use of antifungal drugs have a certain positive effect on the prevention and control of fungal 
infection, there are still some unsolved problems in clinical practice, such as insufficient evidence of some etiology and drug resistance.

Both innate immunity and adaptive immunity play an indispensable role in anti-fungal infection. Mast cells (MCs) are one of the most important innate immune cells, which can initiate and regulate innate and adaptive immunity. Traditionally, MCs have been referred as the key effector cells of allergic diseases, such as urticaria, allergic rhinitis and bronchial asthma (5-7). MCs are mostly located in the border between host and environment such as skin, gastrointestinal and respiratory mucosa and thus may have easy contact with the external environmental pathogens (8). In addition, MCs express a variety of different pattern recognition receptors (PRRs) on their cell surface to detect bacterial, viral, fungal or parasitic components known as pathogen-associated molecular patterns (PAMPs) (9) such as $\beta$-glucan, mannan and lipopolysaccharide. Due to their strategic location and the wide variety of PRRs they express, many researchers have attempted to explore the potential immune relationship between MCs and fungal infection (9-11). Therefore, we will review the role of MCs in fungal infections from molecular mechanism to signaling pathways, and illustrate the role of MCs in some common fungal infections (e.g., Candida albicans (C. albicans), Aspergillus fumigatus (A. fumigatus) and Sporothrix schenckii (S. schenckii)) to light new ideas for prevention and treatment.

\section{FUNGI RECOGNITION RECEPTORS ON MCs}

MCs can directly recognize PAMPs via their PRRs and then be activated. PRRs have comprised mainly five families including Toll-like receptors (TLRs), C-type lectin-like receptors (CLRs), Nucleotide-binding oligomerization domain (NOD)-like receptors (NLRs) and retinoic acid-inducible gene I (RIG-I) like receptors (RLRs) and absent-in-melanoma (AIM)-like receptors (ALRs) (12). Current studies have shown that TLRs and CLRs are main PRRs in host anti-fungal infection. The fungal wall is composed of many layers. Any component of the cell wall could be a potential PAMP. It is reported that the main molecules that trigger the immune response are chitin, $\beta$-glucan, mannan and others (13).

\section{Toll-Like Receptors (TLRs)}

The TLR family is an important group of receptors that recognize invading microorganisms through innate immunity. A total of 13 members of the TLRs family have been reported, 10 of which are associated with human including TLR1-10 (14). TLRs are distributed on a variety of cells including macrophages, dendritic cells and MCs. Mouse connective tissue-type MCs (CTMC) express TLR2, TLR4, and TLR5 on cell surface membrane, whereas TLR3, TLR7, and TLR9 are present both on the cell membrane and intracellularly (15). TLRs can recognize and be activated by different PAMPs. Compared with bone marrow-derived MCs (BMMCs) from wild type mice $\mathrm{C} 57 \mathrm{BL} / 6$, a decrease in $C$. albicans phagocytosis and nitric oxide (NO) production were detected in $\mathrm{TLR}^{-/-}$mice (16). Whereas mannan, extracted from Saccharomyces cerevisiae, directly activates murine CTMC to initiate the proinflammatory response via TLR4 (17). When pretreated with TAK242, a smallmolecule-specific inhibitor of TLR4 signaling, CTMC significantly decreased the generation of cysLT and reactive oxygen species (ROS) as compared to nontreated MCs in response to mannan (17). In summary, TLR2 and TLR4 are the main members of TLRs on MCs with potential to bind different PAMPs on the surface of fungi and induce subsequent host immune response.

\section{C-Type Lectin-Like Receptors (CLRs)}

CLRs are a family of calcium dependent receptors that bind to carbohydrates. A total of 17 members have been reported. Lectin activity of these receptors depends on conserved carbohydrate recognition domains. CLRs include Dectin-1, Dectin-2, macrophage-inducible C-type lectin (Mincle), intercellular adhesion molecule (ICAM-3) and dendritic cell-specific ICAM3-grabbing non-integrin (DC-SIGN) (18). Studies on CLR expression and immune function in MCs are still limited. Dectin-1 is the most commonly studied receptor in CLRs, which exists in both human and murine MCs. Dectin-1 can recognize $\beta$-glucans and trigger a variety of cellular responses such as phagocytosis and the production of cytokines. Zymosan, a $\beta$ glucan derived from yeast of Saccharomyces cerevisiae, for example, induced mature mice peritoneal MCs migration and pro-inflammatory expression via Dectin-1 (19). Similar results were observed in human cord blood-derived mast cells (CBMCs). When incubated with zymosan, CBMCs significantly decreased the level of leukotriene (LT) $\mathrm{B}_{4}$ and $\mathrm{C}_{4}$ in the presence of inhibitors of Dectin-1 (20). Some studies have shown that Dectin-2 can activate FcR $\gamma$ chain to recognize $\alpha$-mannose and induce immune response in dendritic cells (21), macrophages (22), and neutrophils (23), which leads to upregulation of TNF- $\alpha$ and IL-1ra secretion (24). Host Dectin-2 preferentially binds to hyphae form of C. albicans, Microsporum audouinii and Trichophyton rubrum (24), which is essential for inducing Th1/Th17 immune responses (25). The expression and immune function of Dectin-2 of MCs remains to be further studied.

\section{SIGNALING PATHWAYS OF MCs AGAINST FUNGI}

\section{TLR Signaling Pathway}

TLR signaling pathway can be mainly divided into two different mechanisms, namely Myeloid differentiation 88 (MyD88) -dependent pathway and MyD88-independent pathway. The MyD88 dependent pathway is used by all TLRs except TLR3 (26). $\mathrm{MyD}^{-/ /}$mice showed an increase of susceptibility to $C$. albicans infection and the production of proinflammatory cytokines such as TNF- $\alpha$, IFN- $\gamma$ and IL-12p70 could not be detected from antigen-stimulated splenocytes (27). Upon 
activation, MyD88 might trigger several different intracellular signaling pathways such as nuclear factor kappa-B (NF- $\kappa \mathrm{B})$, mitogen activated protein kinases (MAPK including extracellular signal-regulated kinase (ERK), p38 and c-Jun N-terminal kinase (JNK)) and phosphatidylinositol-3 kinase (PI3K), leading to the release of inflammatory cytokines and other inflammatory mediators. IgE-sensitized BMMCs from both TLR2 and MyD88 gene-deleted mice showed a decrease of IL-6 production in comparison of wild type BMMCs (28). Furthermore, a high concentration of Malassezia sympodialis extract inhibited phosphorylation of ERK1/2 and the release of IL-6 in IgE-sensitized BMMCs, indicating that the ERK1/2 pathway may regulate release of IL-6 (28). Except for ERK1/2, other molecules which involved MyD88-dependent pathways for TLR activation have been less investigated in MCs against fungi. PI3K pathway has been reported to be involved in lipopolysaccharide stimulation of murine BMMCs to produce IL-6 and TNF- $\alpha$ (29). In other cells, such as oral epithelial cells, all Candida species were able to activate the NF- $\mathrm{KB}$ and c-Jun pathway and only Candida dubliniensis and C. albicans hyphae can trigger MAPK/MKP1/c-Fos pathway (30).

\section{CLR Signaling Pathway}

CLR signaling pathway can be divided into tyrosine kinase Sykdependent and -independent signaling pathways. Both signaling pathways eventually activated NF- $\mathrm{KB}$, and produced cytokines and chemokines such as IL-6, IL-23, TNF- $\alpha$, macrophage inflammatory protein and CXCL12, which affected differentiation of Th1 and Th17 cells. Traditionally, CLRs (e.g., Dectin-1, Dectin-2) are coupled with Syk to further activate NF$\kappa \mathrm{B}$ pathway by activating caspase recruitment domain protein 9 (CARD9) in Syk-dependent signaling pathway. CARD9 was expressed on the surface of MCs, but its expression level was slightly lower than that of macrophages, dendritic cells and neutrophils. It was found that LTC4 and LTB4 were released from human MCs co-cultured with zymosan, suggesting that the expression of LTC4 and LTB4 was mediated by Dectin-1/ Syk pathway (20). In Syk-independent signaling pathway, activated CLRs (e.g., Dectin-1 and DC-SIGN) can recruit GTPase Ras proteins which subsequently activate serine/ threonine-protein kinase Raf- 1 . This results in Raf-1 mediated phosphorylation of the $\mathrm{p} 65$ subunit of NF- $\mathrm{KB}$, which leads to the production of cytokines (31). In addition, the interaction between PRR can be more effective in the control of fungal infection. Studies have been proved that Dectin-1 and TLR2 collaborate to enhance the role of MAPK pathway resulting in an increase of cytokines (32).

\section{MCs AND FUNGI}

\section{MCs and C. albicans Infection}

C. albicans is one of the most common dimorphic fungus colonizing mucosal surfaces such as gastrointestinal tract,oral-nasal cavity and skin. Vulvovaginal candidiasis is one of the most common forms of candidiasis. When host immunity is suppressed or damaged,
C. albicans can result in severe invasive diseases. Invasive candidiasis is the most common critical care-associated fungal infection in patients hospitalized in intensive care units, with mortality rates between $40 \%$ and $55 \%$ (33).

MCs play a positive role in the defense mechanism against $C$. albicans infections, including phagocytosis and killing yeasts in the extracellular environment (34). Both yeast and hyphae of $C$. albicans can induce the degranulation of BMMCs and lead to the production of different cytokines and chemokines (CCL3 and CCL4) that regulate immune response (35). In response to the stimulation by yeast cells, BMMCs showed increased production of IL- 6 and IL- $\beta$ that could not be induced by hyphae (35). C. albicans hyphae hide their $\beta$-glucan residues beneath a cover of mannoproteins on the cell walls, thus escaping Dectin-1-mediated phagocytosis and stimulating different cytokines production (36). Dectin-1, but not TLR2, is involved in the release of TNF- $\alpha$ through NF-кB/Syk pathway (35). The production of ROS and NO enhanced the ability to defense against $C$. albicans by MCs. When challenged with $C$. albicans, deletion of TLR2 or Dectin-1 significantly reduced NO levels, in comparison with wild type BMMCs. Whether Dectin-1 participates in the production of ROS remains controversial $(16,36)$.

Human MC immune responses towards C. albicans can be mainly divided into three phases. Human mast cell line HMC-1 degranulate in an early and direct response to fungal encounter, thus reducing the viability of C. albicans by $30 \%$ (37). Next, infected cell line HMC-1 release pro-inflammatory cytokines (e.g. IL-8) to recruit neutrophils, followed by the release of antiinflammatory mediators, such as IL-16 and IL-1ra (37). MCs extracellular traps will be formed to contribute to fungal physical restriction, but not killing activity, as there was no significant difference in fungal viability in the presence or absence of nuclease (37). C. albicans can cause MCs death by different mechanisms, including the release of MC extracellular traps and rupture by the growth of internalized C. albicans hyphae, leading to a temporary immune response (37).

Although secretion of monocyte chemotactic protein 1 was not observed in infected MCs (37), they can produce short-lived soluble mediators to improve the crawling of tissue-resident macrophages and induce migration to $C$. albicans infection (38). Uninfected MCs were found to restrict macrophage phagocytosis of C. albicans, which is the result of maintaining a balance between the host and the fungus (38).

MCs exhibited dual effects in response to C. albicans, which is dependent on their protease content and site of $C$. albicans colonization. In mice, two types of MCs have been described as CTMCs, which originate from fetal liver progenitors and are mainly located in stromal tissue, and mucosal MCs (MMCs), which originate in the bone marrow and reside in the intestine and lung (39). Two similar types of MCs are also observed in humans (40). Different MCs types regulated by cytokine production (e.g. TGF- $\beta$, IL-9, IL-10) can discriminate between the fungal morphotypes, thereby promoting local inflammation or protective tolerance. Some studies have found that MMCs contribute to barrier function loss in leaky gut models (39) or higher sensitization against food antigen (41) in response to yeast 
stimulation. Whereas CTMCs induce local protective tolerance to infection by release of anti-inflammatory cytokine in response to hyphae (39). MCs promoting either inflammatory dysbiosis or tolerance were also observed in vulvovaginal candidiasis (42).

\section{MCs and $A$. fumigatus Infection}

A. fumigatus is the most frequent opportunistic pathogenic species of the genus Aspergillus by far, responsible for about $90 \%$ of the cases of Aspergillus diseases (43). A. fumigatus is abundant in the environment, releasing spores (conidia) which are inhaled. After inhalation, patients with lung function or immune defenses impaired are unable to eliminate conidia which will germinate into hyphae that colonize the airways. A. fumigatus hyphae will penetrate pulmonary tissues causing invasive aspergillosis, the most severe disease caused by Aspergillus spp (44). A. fumigatus is known to contain 23 antigenic components that can induce the production of specific IgE (45). Classically, antigen-specific IgE which cross links FceRI can trigger MCs degranulation and increase IL-5 release (46). IL-5 is one of the important cytokines that can promote eosinophils migration to inflammation site, which are associated with severe asthma and allergic bronchial pulmonary aspergillosis $(47,48)$.With further studied, investigators found that mature $A$. fumigatus hyphae can directly stimulate MCs to degranulate in the absence of $\operatorname{IgE}$ (49). When cultivated with heat-killed A. fumigatus spores, HMC-1 cell line was activated to degranulation and release IL-5 (50). Taken together, the activation of MCs induced by $A$. fumigatus may be the initiator of the whole sensitization process. The studies concerning the signaling pathway of immune response of MCs in A. fumigatus infection are scarce. In dendritic cells, TLR2/ MyD88 signaling pathway has been proved to regulate immune response to A. fumigatus conidia, which results in a development of Th2 response (51).

\section{MCs and S. schenckii Infection}

$S$. schenckii is a common biphasic fungal pathogen. The incidence of sporotrichosis is increasing and has become an urgent global health problem (52). The World Health Organization classifies sporotrichosis as one of the Neglected Tropical Diseases which place a significant burden on individuals, families and society (53).

Studies have shown that MCs play a non-protective role in response to $S$. schenckii infection. In our previous study, we

\section{REFERENCES}

1. Wexler AG, Goodman AL. An Insider's Perspective: Bacteroides as a Window Into the Microbiome. Nat Microbiol (2017) 2:17026. doi: 10.1038/ nmicrobiol.2017.26

2. Souza L, Nouér SA, Morales H, Simões B, Solza C, Queiroz-Telles F, et al. Epidemiology of Invasive Fungal Disease in Haematologic Patients. Mycoses (2021) 64(3):252-6. doi: 10.1111/myc.13205

3. Ferrarese A, Cattelan A, Cillo U, Gringeri E, Russo FP, Germani G, et al. Invasive Fungal Infection Before and After Liver Transplantation. World J Gastroenterol (2020) 26(47):7485-96. doi: 10.3748/wjg.v26.i47.7485

4. Wu L, Xiao J, Song Y, Gao G, Zhao H. The Clinical Characteristics and Outcome of Cryptococcal Meningitis With AIDS in a Tertiary Hospital in China: An Observational Cohort Study. BMC Infect Dis (2020) 20(1):912. doi: 10.1186/s12879-020-05661-9 observed that MC-deficient $\mathrm{C} 57 \mathrm{BL} / 6-\mathrm{Kit}^{\mathrm{W}} / \mathrm{Kit}^{\mathrm{W} / \mathrm{v}}$ and $\mathrm{MC}$ deficient Cpa3-Cre/Mcl-1 ${ }^{\mathrm{fl} / \mathrm{fl}}$ (Hello Kitty, HK) mice had more serious inflammation symptoms with elevation of TNF- $\alpha$ and IL-6 in comparison with wild type mice (54). Rat peritoneal MCs can be activated by $S$. schenckii yeasts and release IL- 6 and TNF$\alpha$ via ERK pathway without degranulation $(55,56)$.

\section{DISCUSSION}

MCs are recognized as important effector in response to fungal infection. Their impacts can be protective and non-protective, depending on the pathogen and invasion site. Fungal recognition receptors commonly studied on MCs are TLR2/4 and Dectin1.Upon activated by TLR/MyD88 or Syk pathway, MCs can produce specific cytokines and chemokines which can elicit a direct temporary immune response and recruit some neutrophils to the infection sites. MCs activation can enhance the immune response against infection. In contrast, the involvement of MCs in some immune responses may lead to more severe symptoms, such as intestinal barrier function loss, development of allergic bronchial pulmonary aspergillosis and increased area of inflammatory in S. schenckii infection, which suggests that MCs and their singling pathways are potential treatment regimens to prevent the clinically unwanted consequences. However, up to now, we still have a relatively poor understanding of the mechanism of MCs in response to common fungal infection. Many of the key signaling pathways involved in mediating selective cytokine and chemokine responses from MCs remain unknown. In the future, more in vitro and in vivo studies are urgently needed to further explore the role of $\mathrm{MCs}$ in response to fungal infection.

\section{AUTHOR CONTRIBUTIONS}

MY: substantial contributions to draft the manuscript. X-tS: drafted the article. BL: drafted the article. T-tL: participated in material collection. S-lL: participated in material collection. Z-tZ: substantial contributions to conception and reviewed the article critically for important intellectual content. All authors contributed to the article and approved the submitted version.

5. Song XT, Chen YD, Yu M, Liu B, Zhao ZT, Maurer M. Omalizumab in Children and Adolescents With Chronic Urticaria: A 16-Week Real-World Study. Allergy (2021) 76(4):1271-73. doi: 10.1111/all.14686

6. Chen YD, Maurer M, Yu M, Tu P, Zhao ZT. Addition of Omalizumab to Antihistamine Treatment in Chronic Urticaria: A Real-World Study in China. Ann Allergy Asthma Immunol (2020) 125(2):217-9. doi: 10.1016/j.anai.2020.04.026

7. Chen YD, Krause K, Tu P, Zhao ZT, Maurer M. Response of Omalizumab in Normocomplementemic Urticarial Vasculitis. J Allergy Clin Immunol Pract (2020) 8(6):2114-7.e2. doi: 10.1016/j.jaip.2020.02.024

8. Reber LL, Sibilano R, Mukai K, Galli SJ. Potential Effector and Immunoregulatory Functions of Mast Cells in Mucosal Immunity. Mucosal Immunol (2015) 8(3):444-63. doi: 10.1038/mi.2014.131

9. Jiao Q, Luo Y, Scheffel J, Zhao Z, Maurer M. The Complex Role of Mast Cells in Fungal Infections. Exp Dermatol (2019) 28(7):749-55. doi: 10.1111/ exd.13907 
10. Piliponsky AM, Acharya M, Shubin NJ. Mast Cells in Viral, Bacterial, and Fungal Infection Immunity. Int J Mol Sci (2019) 20(12):2851. doi: 10.3390/ ijms 20122851

11. Bird JA, Sánchez-Borges M, Ansotegui IJ, Ebisawa M, Ortega Martell JA. Skin as an Immune Organ and Clinical Applications of Skin-Based Immunotherapy. World Allergy Organ J (2018) 11(1):38. doi: 10.1186/ s40413-018-0215-2

12. Agier J, Pastwińska J, Brzezińska-Błaszczyk E. An Overview of Mast Cell Pattern Recognition Receptors. Inflamm Res (2018) 67(9):737-46. doi: 10.1007/s00011-018-1164-5

13. García-Carnero LC, Martínez-Álvarez JA, Salazar-García LM, Lozoya-Pérez NE, González-Hernández SE, Tamez-Castrellón AK. Recognition of Fungal Components by the Host Immune System. Curr Protein Pept Sci (2020) 21 (3):245-64. doi: 10.2174/1389203721666191231105546

14. Satoh T, Akira S. Toll-Like Receptor Signaling and Its Inducible Proteins. Microbiol Spectr (2016) 4(6):1-7. doi: 10.1128/microbiolspec.MCHD-0040-2016

15. Agier J, Żelechowska P, Kozłowska E, Brzezińska-Błaszczyk E. Expression of Surface and Intracellular Toll-like Receptors by Mature Mast Cells. Cent Eur J Immunol (2016) 41(4):333-8. doi: 10.5114/ceji.2016.65131

16. Pinke KH, Lima HG, Cunha FQ, Lara VS. Mast Cells Phagocyte Candida Albicans and Produce Nitric Oxide by Mechanisms Involving TLR2 and Dectin-1. Immunobiol (2016) 221(2):220-7. doi: 10.1016/j.imbio.2015.09.004

17. Żelechowska P, Brzezińska-Błaszczyk E, Różalska S, Agier J, Kozłowska E. Mannan Activates Tissue Native and IgE-sensitized Mast Cells to Proinflammatory Response and Chemotaxis in TLR4-dependent Manner. J Leukoc Biol (2021) 109(5):931-42. doi: 10.1002/JLB.4A0720-452R

18. Mayer S, Raulf MK, Lepenies B. C-Type Lectins: Their Network and Roles in Pathogen Recognition and Immunity. Histochem Cell Biol (2017) 147(2):22337. doi: 10.1007/s00418-016-1523-7

19. Żelechowska P, Brzezińska-Błaszczyk E, Różalska S, Agier J, Kozłowska E. Native and IgE-primed Rat Peritoneal Mast Cells Exert Pro-Inflammatory Activity and Migrate in Response to Yeast Zymosan Upon Dectin-1 Engagement. Immunol Res (2021) 69(2):176-88. doi: 10.1007/s12026-02109183-7

20. Olynych TJ, Jakeman DL, Marshall JS. Fungal Zymosan Induces Leukotriene Production by Human Mast Cells Through a dectin-1-dependent Mechanism. J Allergy Clin Immunol (2006) 118(4):837-43. doi: 10.1016/j.jaci.2006.06.008

21. Ishikawa $\mathrm{T}$, Itoh $\mathrm{F}$, Yoshida $\mathrm{S}$, Saijo $\mathrm{S}$, Matsuzawa $\mathrm{T}$, Gonoi $\mathrm{T}$, et al. Identification of Distinct Ligands for the C-type Lectin Receptors Mincle and Dectin-2 in the Pathogenic Fungus Malassezia. Cell Host Microbe (2013) 13(4):477-88. doi: 10.1016/j.chom.2013.03.008

22. Ifrim DC, Quintin J, Courjol F, Verschueren I, van Krieken JH, Koentgen F, et al. The Role of Dectin-2 for Host Defense Against Disseminated Candidiasis. J Interferon Cytokine Res (2016) 36(4):267-76. doi: 10.1089/ jir.2015.0040

23. Wu SY, Weng CL, Jheng MJ, Kan HW, Hsieh ST, Liu FT, et al. Candida Albicans Triggers NADPH Oxidase-Independent Neutrophil Extracellular Traps Through Dectin-2. PloS Pathog (2019) 15(11):e1008096. doi: 10.1371/ journal.ppat.1008096

24. Sato K, Yang XL, Yudate T, Chung JS, Wu J, Luby-Phelps K, et al. Dectin-2 is a Pattern Recognition Receptor for Fungi That Couples With the Fc Receptor Gamma Chain to Induce Innate Immune Responses. J Biol Chem (2006) 281 (50):38854-66. doi: 10.1074/jbc.M606542200

25. Robinson MJ, Osorio F, Rosas M, Freitas RP, Schweighoffer E, Gross O, et al. Dectin-2 is a Syk-coupled Pattern Recognition Receptor Crucial for Th17 Responses to Fungal Infection. J Exp Med (2009) 206(9):2037-51. doi: $10.1084 / \mathrm{jem} .20082818$

26. Akira S, Takeda K, Kaisho T. Toll-Like Receptors: Critical Proteins Linking Innate and Acquired Immunity. Nat Immunol (2001) 2(8):675-80. doi: $10.1038 / 90609$

27. Villamón E, Gozalbo D, Roig P, Murciano C, O’Connor JE, Fradelizi D, et al. Myeloid Differentiation Factor 88 (MyD88) is Required for Murine Resistance to Candida Albicans and is Critically Involved in Candida-induced Production of Cytokines. Eur Cytokine Netw (2004) 15(3):263-71.

28. Selander C, Engblom C, Nilsson G, Scheynius A, Andersson CL. Tlr2/MyD88dependent and -Independent Activation of Mast Cell IgE Responses by the Skin Commensal Yeast Malassezia Sympodialis. J Immunol (2009) 182 (7):4208-16. doi: 10.4049/jimmunol.0800885
29. Hochdörfer T, Kuhny M, Zorn CN, Hendriks RW, Vanhaesebroeck B, Bohnacker T, et al. Activation of the PI3K Pathway Increases TLR-induced Tnf- $\alpha$ and IL-6 But Reduces IL-1 $\beta$ Production in Mast Cells. Cell Signal (2011) 23(5):866-75. doi: 10.1016/j.cellsig.2011.01.012

30. Moyes DL, Murciano C, Runglall M, Kohli A, Islam A, Naglik JR. Activation of MAPK/c-Fos Induced Responses in Oral Epithelial Cells is Specific to Candida Albicans and Candida Dubliniensis Hyphae. Med Microbiol Immunol (2012) 201(1):93-101. doi: 10.1007/s00430-011-0209-y

31. Gringhuis SI, den Dunnen J, Litjens M, van der Vlist M, Wevers B, Bruijns SC, et al. Dectin-1 Directs $\mathrm{T}$ Helper Cell Differentiation by Controlling Noncanonical NF-kappaB Activation Through Raf-1 and Syk. Nat Immunol (2009) 10(2):203-13. doi: 10.1038/ni.1692

32. Goodridge HS, Underhill DM. Fungal Recognition by TLR2 and Dectin-1. Handb Exp Pharmacol (2008) 183):87-109. doi: 10.1007/978-3-540-721673_5

33. Logan C, Martin-Loeches I, Bicanic T. Invasive Candidiasis in Critical Care: Challenges and Future Directions. Intensive Care Med (2020) 46(11):2001-14. doi: $10.1007 / s 00134-020-06240-\mathrm{x}$

34. Trevisan E, Vita F, Medic N, Soranzo MR, Zabucchi G, Borelli V. Mast Cells Kill Candida Albicans in the Extracellular Environment But Spare Ingested Fungi From Death. Inflammation (2014) 37(6):2174-89. doi: 10.1007/s10753014-9951-9

35. Nieto-Patlán A, Campillo-Navarro M, Rodríguez-Cortés O, Muñoz-Cruz S, Wong-Baeza I, Estrada-Parra S, et al. Recognition of Candida Albicans by Dectin-1 Induces Mast Cell Activation. Immunobiol (2015) 220(9):1093-100. doi: 10.1016/j.imbio.2015.05.005

36. Yang Z, Marshall JS. Zymosan Treatment of Mouse Mast Cells Enhances Dectin-1 Expression and Induces dectin-1-dependent Reactive Oxygen Species (ROS) Generation. Immunobiology (2009) 214:321-30. doi: 10.1016/ j.imbio.2008.09.002

37. Lopes JP, Stylianou M, Nilsson G, Urban CF. Opportunistic Pathogen Candida Albicans Elicits a Temporal Response in Primary Human Mast Cells. Sci Rep (2015) 5:12287. doi: 10.1038/srep12287

38. De Zuani M, Paolicelli G, Zelante T, Renga G, Romani L, Arzese A, et al. Mast Cells Respond to Candida Albicans Infections and Modulate Macrophages Phagocytosis of the Fungus. Front Immunol (2018) 9:2829. doi: 10.3389/ fimmu.2018.02829

39. Renga G, Moretti S, Oikonomou V, Borghi M, Zelante T, Paolicelli G, et al. IL9 and Mast Cells Are Key Players of Candida Albicans Commensalism and Pathogenesis in the Gut. Cell Rep (2018) 23(6):1767-78. doi: 10.1016/ j.celrep.2018.04.034

40. Church MK, Kolkhir P, Metz M, Maurer M. The Role and Relevance of Mast Cells in Urticaria. Immunol Rev (2018) 282(1):232-47. doi: 10.1111/imr.12632

41. Yamaguchi N, Sugita R, Miki A, Takemura N, Kawabata J, Watanabe J, et al. Gastrointestinal Candida Colonisation Promotes Sensitisation Against Food Antigens by Affecting the Mucosal Barrier in Mice. Gut (2006) 55(7):954-60. doi: 10.1136/gut.2005.084954

42. Renga G, Borghi M, Oikonomou V, Mosci P, Bartoli A, Renauld JC, et al. Il-9 Integrates the Host-Candida Cross-Talk in Vulvovaginal Candidiasis to Balance Inflammation and Tolerance. Front Immunol (2018) 9:2702. doi: 10.3389/fimmu.2018.02702

43. Strickland AB, Shi M. Mechanisms of Fungal Dissemination. Cell Mol Life Sci (2021) 78(7):3219-38. doi: 10.1007/s00018-020-03736-Z

44. Gomez C, Carsin A, Gouitaa M, Reynaud-Gaubert M, Dubus JC, Mège JL, et al. Mast Cell Tryptase Changes With Aspergillus Fumigatus - Host Crosstalk in Cystic Fibrosis Patients. J Cyst Fibros (2018) 17(5):631-5. doi: 10.1016/j.jcf.2018.01.012

45. Fukutomi Y, Taniguchi M. Sensitization to Fungal Allergens: Resolved and Unresolved Issues. Allergol Int (2015) 64(4):321-31. doi: 10.1016/ j.alit.2015.05.007

46. Mathias CB, Freyschmidt EJ, Caplan B, Jones T, Poddighe D, Xing W, et al. Ige Influences the Number and Function of Mature Mast Cells, But Not Progenitor Recruitment in Allergic Pulmonary Inflammation. J Immunol (2009) 182(4):2416-24. doi: 10.4049/jimmunol.0801569

47. Morán G, Uberti B, Ortloff A, Folch H. Aspergillus Fumigatus-Sensitive IgE is Associated With Bronchial Hypersensitivity in a Murine Model of Neutrophilic Airway Inflammation. J Mycol Med (2018) 28(1):128-36. doi: 10.1016/j.mycmed.2017.11.005 
48. Wu G, Meng X, Zheng P, Zhang XD, Li L, Hu H, et al. Elevated Serum Levels of Periostin in Patients With Allergic Bronchopulmonary Aspergillosis. Mycoses (2019) 62(9):780-9. doi: 10.1111/myc.12957

49. Urb M, Pouliot P, Gravelat FN, Olivier M, Sheppard DC. Aspergillus Fumigatus Induces Immunoglobulin E-independent Mast Cell Degranulation. J Infect Dis (2009) 200(3):464-72. doi: 10.1086/600070

50. Chen LS, Li YM, Tong XL, Zhao ZT, Wang C. [Effects of Aspergillus Fumigatus Spore on the Activation of Mast Cell]. Chin J Mycol (2017) 12 (1):3-7. doi: 10.3969/j.issn.1673-3827.2017.01.002

51. Percier P, De Prins S, Tima G, Beyaert R, Grooten J, Romano M, et al. Aspergillus Fumigatus Recognition by Dendritic Cells Negatively Regulates Allergic Lung Inflammation Through a TLR2/MyD88 Pathway. Am J Respir Cell Mol Biol (2021) 64(1):39-49. doi: 10.1165/rcmb.2020-0083OC

52. Lopes-Bezerra LM, Mora-Montes HM, Zhang Y, Nino-Vega G, Rodrigues AM, de Camargo ZP, et al. Sporotrichosis Between 1898 and 2017: The Evolution of Knowledge on a Changeable Disease and on Emerging Etiological Agents. Med Mycol (2018) 56(suppl_1):126-43. doi: 10.1093/ mmy/myx103

53. Organization WH. Working to Overcome the Global Impact of Neglected Tropical Diseases: First WHO Reporton Neglected Tropical Diseases: Summary[J]. Geneva Switzerland Who (2011) 7(4244):596.
54. Jiao Q, Luo Y, Scheffel J, Geng P, Wang Y, Frischbutter S, et al. Skin Mast Cells Contribute to Sporothrix Schenckii Infection. Front Immunol (2020) 11:469. doi: 10.3389/fimmu.2020.00469

55. Romo-Lozano Y, Hernández-Hernández F, Salinas E. Mast Cell Activation by Conidia of Sporothrix Schenckii: Role in the Severity of Infection. Scand J Immunol (2012) 76(1):11-20. doi: 10.1111/j.1365-3083.2012.02706.x

56. Romo-Lozano Y, Hernández-Hernández F, Salinas E. Sporothrix Schenckii Yeasts Induce ERK Pathway Activation and Secretion of IL-6 and TNF- $\alpha$ in Rat Mast Cells, But No Degranulation. Med Mycol (2014) 52(8):862-8. doi: $10.1093 / \mathrm{mmy} / \mathrm{myu} 055$

Conflict of Interest: The authors declare that the research was conducted in the absence of any commercial or financial relationships that could be construed as a potential conflict of interest.

Copyright (c) $2021 \mathrm{Yu}$, Song, Liu, Luan, Liao and Zhao. This is an open-access article distributed under the terms of the Creative Commons Attribution License (CC BY). The use, distribution or reproduction in other forums is permitted, provided the original author(s) and the copyright owner(s) are credited and that the original publication in this journal is cited, in accordance with accepted academic practice. No use, distribution or reproduction is permitted which does not comply with these terms. 